\title{
Comparison of the Pain Relief Management of Acute Post- Operative Pain in Two Surgical Units in Hungary
}

\author{
Csilla A. Reszegi ${ }^{1}$, Tamás Szabó², Andrea Meskó ${ }^{1}$, Romána Zelkó ${ }^{* 1}$ and Balázs Hankó ${ }^{1}$ \\ ${ }^{1}$ University Pharmacy Department of Pharmacy Administration, Semmelweis University, Högyes Endre Str. 7- \\ 9., $\mathrm{H}$-1092 Budapest, Hungary \\ ${ }^{2}$ Toldy Ferencz Hospital, Törteli Street 1-3, H-2700 Cegléd, Hungary
}

\begin{abstract}
The purpose of the study is to review the administration of pain relief medications in acute post-operative phase and compare the drug therapy after major surgical interventions in inpatient care units in order to assure quality of pain management.

A double-group comparative study design was chosen using observations as comparison of examining pain regimens in two surgical units by comparing the 4-year recorded post-operative drug regimens of a total of 1054 patients after major surgical interventions; hip and knee replacements in Traumatology and the post-operative drug regimen administered following major surgical intervention at the department of Gynaecology, respectively.

The results of the study suggest significant difference in medications, and dosage forms of medications used for acute post-operative pain relief through 4 consecutive years at Traumatology and Gynaecology. At the in patient unit of Traumatology the administration of Tramadol hydrochloride droplets has significantly increased while at Gynaecology the use of Nalbuphine hydrochloride ampoules showed significant increase. The results indicate that pain relief, even in acute therapy, requires effective and accurately administered doses. The latter, as this study points out, could be successfully solved with the conventional droplet dosage form. Inter-professional collaboration of doctors, clinical pharmacists and nurses has an impact to provide patients with successful, adequate and individually customized pain relief methods.
\end{abstract}

Keywords: Post-operative pain management, Inter-professional collaboration, Acute Pain Therapy, Dosage form.

\section{INTRODUCTION}

Undermanaged post-operative pain remains a widespread problem despite the development of specialized acute pain regimens over the past 20 years [1, 2]. A major review [3] and a systematic review [4] similarly confirm the difficulties of providing effective post-operative pain management to large populations of surgical patients; $70-90 \%$ of the cases the care provided is inadequate. At the same time the consequences of the under managed post-operative pain are obvious and are preventable by collaboration of inter-professional health care providers in the process of acute post-operative pain management.

Guidelines for managing acute pain exist at a local level in many hospitals, but the published guidelines may be influenced by local factors, such as: ward routines, the available surgical and anaesthetic experience and skills, cultural and social preferences, and the availability of International Guidelines $[5,6]$.

Patients' perspectives on pain management have mainly been addressed with descriptions of their experience [7].

*Address correspondence to this author at the University Pharmacy Department of Pharmacy Administration, Semmelweis University, Högyes Endre Str. 7-9., H-1092 Budapest, Hungary; Tel/Fax: +36-1-2170927;

E-mail: zelrom@gytk.sote.hu
Many post-operative analgesic regimens rely on patients to self-administer analgesics following surgical interventions. For example, a patient may be given a prescription for tablets and told to take one every few hours as needed. The importance of effective pain control in minimizing the deleterious effects of the surgically induced stress response and improving outcome from surgery is now well recognized $[8,9]$.

The view has therefore arisen that such information needs to be procedure-specific, since the analgesic efficacy of drugs varies between different types of surgery: e.g. the analgesic efficacy of paracetamol is twofold less in orthopaedic procedures compared with dental procedures [10]. In addition, the difference in analgesic efficacy between non-steroidal antiinflammatory agents (NSAIDs) and paracetamol has been demonstrated to depend on the magnitude of surgery [11]. Optimal dynamic pain relief, allowing early function, is a prerequisite for accelerated postoperative rehabilitation (fast-track surgery) [8], and therefore the clinician needs procedure-specific information in order to optimize the choice of analgesia. Further supporting a need for procedure-specific information is the fact that the intensity of pain and the consequential effects on organ functions may be procedure-related. The purpose of the study is to review and analyze the administration of pain relief medications in acute post- 
operative phase, compare the drug therapies, including drug forms after major surgical interventions in inpatient care units and point out the roles of interprofessional health care providers, such as pharmacists, in the process of acute post-operative pain management.

\section{METHODS}

\section{Sample Selection}

A retrospective cohort study was carried out based on data analysis from the reporting system of the Toldy Ferencz hospital for the 4-year period from January 2006 through December 2009. These data coded and abstracted from the health record after recovery of surgical interventions is completed, contain demographic and up to 4 diagnosis codes. A sample of individuals is selected to compare pain regimens in two surgical units of a metropolitan hospital of Cegléd, Hungary by comparing the 4-year recorded (Y1-Y4) post-operative drug regimens over a thousand patients (1054). The hospital has 50 acute beds at Traumatology and 50 beds for acute in-patient care at Gynaecology. The total number of beds for acute care at the hospital is 458 (www.OEP.hu, 2009).

In 2009: at the inpatient care department of Traumatology 1718 surgical interventions, including hip and knee surgeries, were performed; and 1974 surgical interventions, including uterus removals, were performed at the inpatient Department of Gynaecology. (http://www.toldykorhaz.hu, 2009).

The 1054 patients have received analgesics, as part of the acute pain management therapy, after major surgical interventions, such as: hip and knee replacements in Traumatology (408 female patients, 291 male patients) and Gynecology (355 female patients) at the department of Toldy Ferencz inpatient care centre.

Figure 1 illustrates the age-distribution of patients at Traumatology after hip and knee replacement interventions, respectively. Knee replacements were chosen because knee is the one most affected by pain, which is exposed to great forces in daily activities and the knee is the most treated anatomical region, being responsible for $26 \%$ of all visits to orthopaedists [12]. The recorded data were stratified according to mode of analgesic delivery and type of agent for the comparison. Figure 2 shows that of the patients after uterus removal at Gynaecology. The samples of the age-distribution of patients (Figures $\mathbf{1}$ and 2) follow a gaussain distribution. Table $\mathbf{1}$ shows the active ingredients of Analgesics, NSAIDs and Opioids that are used in accordance with International Guidelines for Acute Pain Management.

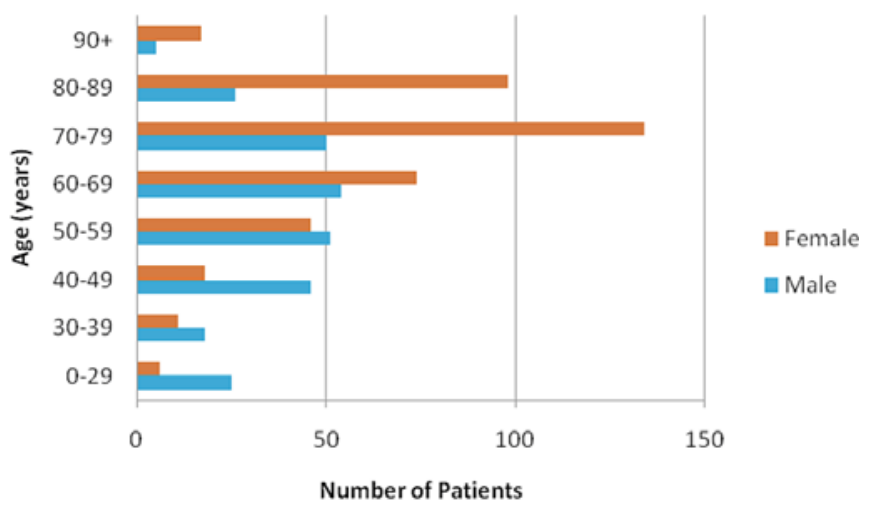

Figure 1: Age distribution of patients treated at Traumatology.

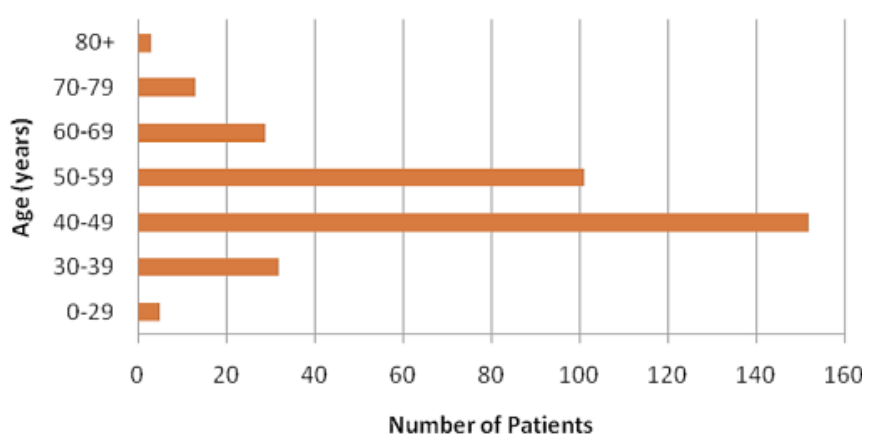

Figure 2: Age distribution of patients treated at Gynecology.

The NSAIDs are a group of chemically heterogeneous compounds with anti-inflammatory, analgesic, and antipyretic effects, excluding acetaminophen, which lacks significant antiinflammatory properties [13].

The term "narcotic", which initially referred to a drug that induces stupor, has been replaced by "opioid" (drugs related to opium), a more appropriate pharmacologic designation for a class of medications used to treat pain. The pharmacodynamic (e.g., potency) and pharmacokinetic (e.g., rate and route of elimination) properties of various agents differ significantly, changes in drug, dosing, and route of administration cannot be carried out without considering equianalgesic equivalents on a drug and dose basis [14, 15]. Equianalgesic guidelines are available which provide information about approximately equivalent doses between drugs and routes of administration [16]. 
Table 1: Analgesics, NSAIDs, and Opioids that are used in accordance with International Guidelines for Acute Pain Management WHO Collaborating Centre for Drug Statistics Methodology, 2003 [16]

\begin{tabular}{|c|c|}
\hline Analgesics and NSAIDs & Opioids \\
\hline \hline acetylsalicylic acid & codeine \\
diclofenac sodium & morphine sulfate \\
ibuprofen & pentazocine \\
indomethacin & pethidine \\
naproxen & tramadol \\
paracetamol & \\
phenylbutazone & \\
\hline
\end{tabular}

\section{Hypothesis Testing}

The chi-square test [17] is used to test if a sample of data came from a population with a specific distribution.

Chi-square test was made for both groups to check whether there are any differences between the years. The null hypothesis was that there is no difference in the post-operative drug regimen administered for subsequent years. The significance level was set at $5 \%$. SPSS 11.5 statistical program package was used for the calculations.

\section{RESULTS AND DISCUSSION}

Figures 3 and 4 illustrate the changes of the number patients treated with analgesics after major surgical interventions in acute post-operative phase as a function of time.

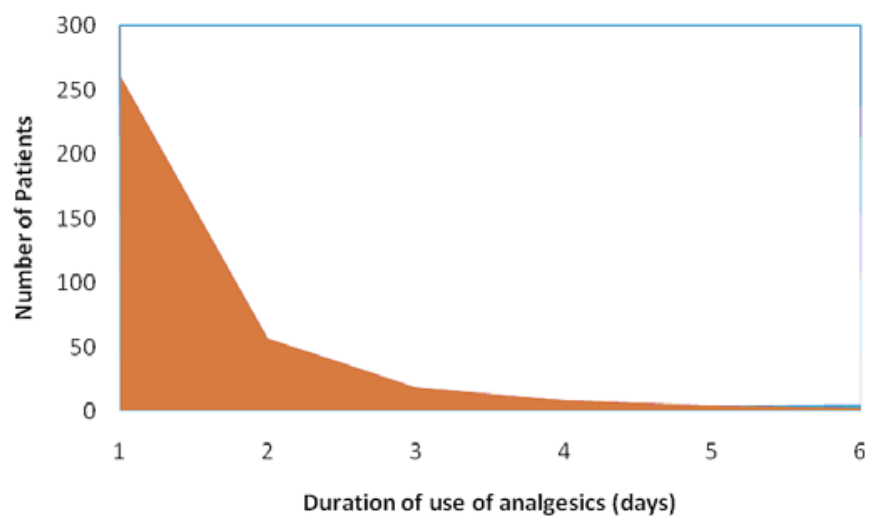

Figure 3: Post-operative active pain relief therapy at Gynaecology as a function of the duration if analgesic use.

Table 2 illustrates the brand names of marketing authorized products, their active ingredients, and the ATC codes of medications used for Acute Pain Relief and were administered from 2006 to 2009 at Traumatology in acute Post-operative Phase, while Table 3 summarizes those were applied within the same period at Gynaecology in acute Post-operative Phase after uterus removal as part of the recovery.

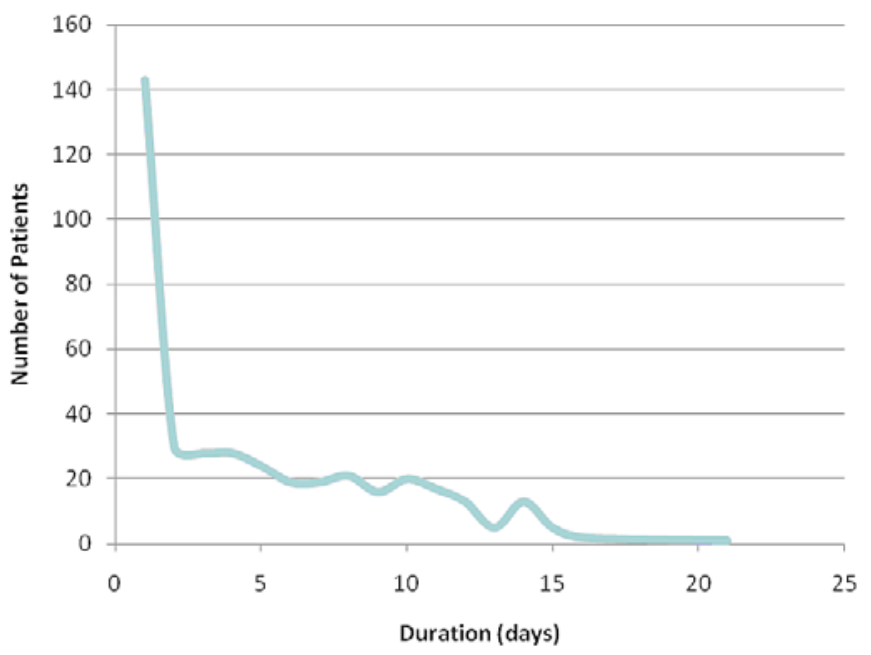

Figure 4: Duration (days) of active pain relief treatment at Traumatology.

Table 2: The Applied Drugs for Acute Pain Relief within 2006-2009 at Traumatology

\begin{tabular}{|c|c|c|}
\hline Brand Name & Active Ingredient & ATC \\
\hline \hline Algopyrin & Metamizole sodium & N02BB02 \\
\hline Cataflam & Diclofenac & M01AB05 \\
\hline Contramal & Tramadol & N02AX02 \\
\hline Demalgonil & $\begin{array}{c}\text { Allobarbital, } \\
\text { aminophenazone }\end{array}$ & N02BB73 \\
\hline Panadol & Paracetamol & N02BE01 \\
\hline Panalgorin & Metamizole sodium & N02BB02 \\
\hline
\end{tabular}

Table 3: The Applied Drugs for Acute Pain Relief within 2006-2009 at Gynaecology

\begin{tabular}{|c|c|c|}
\hline Brand Name & Active Ingredient & ATC \\
\hline \hline Algopyrin & Metamizole sodium & N02BB02 \\
\hline Contramal & Tramadol & N02AX02 \\
\hline Nubain & Nalbuphine & N02AF02 \\
\hline Panalgorin & Metamizole sodium & N02BB02 \\
\hline
\end{tabular}

We have reviewed descriptive statistics for Traumatology and Gynaecology data, separately. Results of the descriptive statistical analysis (including frequency tables and diagrams) show that there are major differences in the types of drugs used in the four 
year period for both groups. These changes do not show systematic differences either in the direction or magnitude over the years (2006-2009).

Table 4 summarizes the results of the descriptive statistics for Traumatology and Gynaecology separately. In fact, only seven of the thirty two drugs are used for all the years for Traumatology and only five of the sixteen drugs for Gynecology. For example, as new drugs are introduced others appear less frequently. The seven drugs that appear in each year in Traumatology include: Algopyrin ampullas, Cataflam capsules, Contramal capsules, Contramal droplets, Demalgonil ampullas, Panadol tablets, Panalgorin tablets* ("marketing authorized products including their dosage forms), while the five drugs that appeared for Gynaecology include: Algopyrin ampullas, Contramal ampullas, Contramal capsules, Nubain ampullas, Panalgorin tablets* ${ }^{*}{ }^{*}$ marketing authorized products including their dosage forms).
Pearson correlation was calculated to assess the relationship between the treatments for the years. The coefficients were minor and not significant. Table $\mathbf{5}$ summarizes the results of the hypothesis testing. Zero hypothesis was that there was no difference in the post-operative drug regimen administered for the four consecutive years (Y1-Y4 referring to 2006-2009) and there is no significant difference among years (2006 to 2007), (2007 to 2008), and (2008 to 2009). The results of the chi-square tests have shown that there have been significant differences. The $p$ value was smaller than 0.001 for all cases.

Significant differences were pronounced among the drugs administered for the four consecutive years. It appears that pure opioid agonist was not given for pain control in the study population.

The appearance of generics could replace the original drugs (i.e.: Cataflam was replaced by Fortedol).

Table 4: Comparison of the Descriptive Statistics for Traumatology and Gynaecology Concerning the Applied Marketing Authorized Medicines

\begin{tabular}{|c|c|c|c|c|c|c|c|c|}
\hline \multirow{2}{*}{$\begin{array}{c}\begin{array}{c}\text { Marketing } \\
\text { authorized } \\
\text { products }\end{array} \\
\text { Brand Names }\end{array}$} & \multicolumn{2}{|c|}{2006} & \multicolumn{2}{|c|}{2007} & \multicolumn{2}{|c|}{2008} & \multicolumn{2}{|c|}{2009} \\
\hline & Gynecol. & Traum. & Gynecol. & Traum. & Gynecol. & Traum & Gynecol. & Traum \\
\hline Algopyrin amp & 0.68 & 0.22 & 1.18 & 0.15 & 0.30 & 0.08 & 0.59 & 0.56 \\
\hline Cataflam tbl & 0.82 & 9.88 & 0.00 & 12.63 & 0.10 & 11.15 & 0.00 & 4.45 \\
\hline Contramal amp & 2.47 & 0.08 & 4.12 & 0.76 & 5.55 & 0.58 & 2.82 & 0.62 \\
\hline $\begin{array}{c}\text { Contramal } \\
\text { droplets }\end{array}$ & 0.00 & 107.00 & 0.00 & 44.37 & 0.00 & 86.17 & 0.00 & 135.44 \\
\hline Contramal caps & 5.21 & 1.38 & 0.39 & 2.55 & 2.15 & 3.64 & 0.12 & 3.94 \\
\hline Demalgonil amp & 0.00 & 0.11 & 0.00 & 0.10 & 0.00 & 0.05 & 0.53 & 0.30 \\
\hline Nubain amp & 0.55 & 0.05 & 1.18 & 0.10 & 3.75 & 0.06 & 8.35 & 0.01 \\
\hline Panadol tbl & 0.00 & 0.02 & 0.59 & 0.15 & 0.00 & 2.02 & 0.18 & 0.34 \\
\hline Panalgorin tbl & 0.55 & 0.30 & 1.76 & 1.35 & 0.70 & 2.22 & 0.59 & 1.50 \\
\hline
\end{tabular}

Gynecol: Gynaecology inpatient care unit; Traum: Traumatology inpatient care unit

Table 5: Results of the Hypothesis Testing

\begin{tabular}{|c|c|c|c|c|c|}
\hline In-patient unit & Years & Chi-square $\left(\mathbf{X}^{2}\right)$ test & Degree of freedom & P value & Results \\
\hline \hline Traumatology & Y1-Y2 & 925.157 & 7 & $p<0.001$ & significant difference \\
\hline & Y2-Y3 & 339.767 & 7 & $\mathrm{p}<0.001$ & significant difference \\
\hline & $\mathrm{Y3}$-Y4 & 822.294 & 7 & $\mathrm{p}<0.001$ & significant difference \\
\hline Gynaecology & $\mathrm{Y} 1$-Y2 & 114.941 & 4 & $\mathrm{p}<0.001$ & significant difference \\
\hline & $\mathrm{Y} 2-Y 3$ & 62.856 & 4 & $\mathrm{p}<0.001$ & significant difference \\
\hline & $\mathrm{Y3}$-Y4 & 52.359 & 4 & $\mathrm{p}<0.001$ & significant difference \\
\hline
\end{tabular}


The proportional application of Fortedol instead of Cataflam was observed. The appearance of Fortedol tablets can be attributed to launching of new generics to the market. The administration of droplet dosage form of Contramal is significantly increased. This conventional droplet dosage form enables accurate dosing due to its equianalgesic property compare to Tramadol capsules. In addition, the oral administration of Contramal droplets, in contrast to the solid dosage forms of the active ingredient of the oral medications, has the advantages of better, more sensitive dosing in pain relief, and better side effect profile due to the possibility of their sensitive, drop-by-drop dosing.

\section{CONCLUSIONS}

The results of this study suggest that the adequate acute post-operative pain relief regimen is the base of quality assurance of pain management and is the essential component of accelerated recovery.

The prescribed and administered drug therapies at both inpatient units are part of and included the Protocol of the Department of Anaesthesiology and Intensive Therapy, Collegium Medium, (http://www. drdiag.hu).

We observed that new methods of administration, delivery systems and dosage forms have evolved over past five years in the area of postoperative pain management. Launching of new generics has impact on the administered pain relief medications as substitutes to the original medications. Providing effective pain control is a central role in postoperative phase to assure accelerated recovery. This study points out that the understanding of the administration of the adequate and effective dosage forms of analgesics is paramount to providing optimal pain relief in acute post-operative pain management. The advantages and also disadvantages, such as: side effect profile of each dosage form must be considered when selecting the best form for administration.

Pharmacists have a significant role to play in collaboration with other health care professional to find the best dosage forms for patients with individual pain relief needs to assure quality of care.

Finally, pain management therapy is part of the postsurgical phase of recovery. Since pharmacists work in inter-professional collaboration with other health care providers, bear a responsibility for therapies used for controlling pain from surgery assure patients' recovery and help the implementation of the best evidence based medicine focusing on adequacy and patient safety.

\section{REFERENCES}

[1] Warfield, C. A. \& Kahn, C. H. (1995). Acute pain management. Programs in U.S. hospitals and experiences and attitudes among U.S. adults. Anesthesiology 83, 10901094. http://dx.doi.org/10.1097/00000542-199511000-00023

[2] Apfelbaum, J. L., Chen, C., Mehta, S. S., \& Gan T. J. (2003) Postoperative pain experience: results from a national survey suggest postoperative pain continues to be undermanaged. Anesthesia and Analgesia 97, 534-540. http://dx.doi.org/10.1213/01.ANE.0000068822.10113.9E

[3] Power, I. (2005). Recent advances in postoperative pain therapy. British Journal of Anaesthesia 95, 43-51. http://dx.doi.org/10.1093/bja/aei037

[4] Dolin, S. J., Cashman, J. N., \& Bland, J. M. (2002). Effectiveness of acute postoperative pain management: I. Evidence from published data. British Journal of Anaesthesia 89, 409-423.

http://dx.doi.org/10.1093/bja/89.3.409

[5] Practice guidelines for acute pain management in the perioperative setting. A report by the American Society of Anesthesiologists Task Force on Pain Management, Acute Pain Section. (1995). Anesthesiology 82, 1071-1081.

[6] Moore, A., Edwards, J., Barden, J., McQuay H. (2003). Bandolier's little book of pain. Oxford: Oxford University Press.

[7] Archibald, G. (2003). Patients' experiences of hip fractures. Journal of Advanced Nursing44, 385-392. http://dx.doi.org/10.1046/j.0309-2402.2003.02817.x

[8] Kehlet, H., \& Dahl, J. B. (2003). Anaesthesia, surgery, and challenges in postoperative recovery. Lancet 362, 19211928.

http://dx.doi.org/10.1016/S0140-6736(03)14966-5

[9] Kehlet, H. (2005). Postoperative opioid sparing to hasten recovery: what are the issues? Anesthesiology; 102, 10831085.

http://dx.doi.org/10.1097/00000542-200506000-00004

[10] Gray, A., Kehlet, H., Bonnet, F., \& Rawal, N. (2005). Predicting postoperative analgesia outcomes: NNT league tables or procedure-specific evidence? British Journal of Anaesthesia 94, 710-714.

http://dx.doi.org/10.1093/bja/aei144

[11] Hyllested, M., Jones, S., Pedersen, J. L., \& Kehlet, H. (2002) Comparative effect of paracetamol, NSAIDs or their combination in postoperative pain management: a qualitative review. British Journal of Anaesthesia 88, 199-214. http://dx.doi.org/10.1093/bja/88.2.199

[12] American Academy of Orthopaedic Surgeons (1997) 6 Million a Year Seek Medical Care for Knees. Rosemont, IL: American Academy of Orthopaedic Surgeons.

[13] Goodman, L.S., Gilman, A., Brunton, L. L., Lazo, J.S., \& Parker, K.L. (2006). Goodman \& Gilman's the pharmacological basis of therapeutics $\left(11^{\text {th }}\right.$ ed.). New York: McGraw-Hill.

[14] American Pain Society (2003). Principles of analgesic use in the treatment of acute pain and cancer pain $\left(5^{\text {th }}\right.$ ed.) Glenview, IL: American Pain Society.

[15] Pasero, C., Portenoy, R.K., \& McCaffery, M (1999). Opioid analgesics, In M., McCaffery, C, (eds). Pasero, Pain: clinica;I manual $\left(2^{\text {nd }}\right.$ ed. $)$, pp. 240-243. St. Louis: Mosby.

[16] Patanwala, A.E., Duby, J., Waters, D., \& Erstad, B.L. (2007) Opiod conversions in acute care. The Annals of 
Pharmacotherapy, 41, 255-266.

http://dx.doi.org/10.1345/aph.1H421
[17] Snedecor and Cochran, 1989. http://www.itl.nist.gov/div/ handbook/eda/section3/eda35f.htm

Received on 22-10-2014

Accepted on 04-11-2014

Published on 31-12-2014

DOI: http://dx.doi.org/10.14205/2311-0317.2014.02.01.2

(C) 2014 Reszegi et al.; Licensee Pharma Publisher.

This is an open access article licensed under the terms of the Creative Commons Attribution Non-Commercial License (http://creativecommons.org/licenses/by-nc/3.0/) which permits unrestricted, non-commercial use, distribution and reproduction in any medium, provided the work is properly cited. 